\title{
The effect of ERAS management in gastric cancer: assessment from the IMIGASTRIC study
}

\author{
Francesco Giovanardi ${ }^{1,2 *}$, Francesco Falbo ${ }^{3}$, Chiara Celano ${ }^{1}$, Michele Casella ${ }^{1}$, Marco Palisi $^{1}$
}

'Department of General and Oncological Surgery, Azienda Ospedaliera Ordine Mauriziano "Umberto I", Turin, Italy.
2Department of General Surgery and Organ Transplantation "Paride Stefanini”, Sapienza University of Rome, Rome, Italy.
${ }^{3}$ Department of Surgical Sciences, Sapienza University of Rome, Rome, Italy.

\section{To Cite}

Giovanardi F, Falbo F, Celano C, Casella M, Palisi M. The effect of ERAS management in gastric cancer: assessment from the IMIGASTRIC study. J Gastric Surg 2020, 2(4).

\section{Publication history}

Received: October 27, 2020

Accepted: November 02, 2020

Article in press: November 03, 2020

Published online: November 05, 2020

\section{* Correspondence to}

Dr. Francesco Giovanardi

Department of General Surgery

and Organ Transplantation "Paride Stefanini",

Sapienza University of Rome.

Viale del Policlinico, 155

00161, Rome, Italy.

giovanardi89@gmail.com

Telephone: +393202137231

\section{ABSTRACT \\ Background:}

Establish protocols to enhance the surgical management (ERAS) can improve outcomes, shortening hospital stay and save resources.

Several studies have carried out for colorectal surgery, while a lack of evidence for gastrectomy remains.

This study aims to evaluate the impact of ERAS strategies in a large series of patients underwent gastric cancer surgery.

Methods:

This is a propensity score-matched case-control study, comparing an ERAS group with a control group. Data were recorded through a tailored and protected web-based system. Primary outcomes: hospital stay, complications rate. Among the secondary outcomes, there are: POD of mobilization, POD of starting liquid diet and soft solid diet.

\section{Results:}

Patients in the ERAS and control groups were 1:1 matched by the closest propensity score on the logit scale and with a Caliber $=0.2$. The successful matching resulted in a total sample of 440 patients.

The two groups showed no differences in all baseline patients characteristics, type of surgery $(\mathrm{P}=0.31)$ and stage of the disease $(\mathrm{P}=0.61)$.

A benefit in favor of the ERAS management was found in the length of hospital stay $(\mathrm{P}=0.0004)$ and complications rate $(\mathrm{P}=0.001)$.

Conclusion:

An ERAS program can safely be established in referral centers for gastric cancer, enabling to significantly improve the main clinical outcomes.

Key Words:

ERAS; Enhanced Recovery After Surgery; gastric cancer; gastric surgery; gastrectomy. 


\section{Background}

Gastric cancer is the second leading cause of cancer death in the world and surgery plays the most important role in the treatment of this disease.

However, surgery for gastric cancer remains a highrisk procedure with clinically significant postoperative stress, complications, and significant sequelae.

Significant advances in the management of surgical patients have been in last decades for gastric cancer.

This has led to the concept of enhanced recovery after surgery (ERAS) with the objective of reducing the length of hospital stay accelerating postoperative recovery and reducing surgical stress.

The ERAS protocols have many items, including the preoperative patient education, preoperative carbohydrate loading, early mobilization and feeding starting from the first postoperative days.

Most of studies on this field were published for colorectal surgery, while only few authors described ERAS protocols in gastric cancer surgery.

In the present study, we have evaluated the effects on postoperative functional recovery outcomes after gastrectomy in patients undergoing an ERAS program in comparison with a conventional surgical management.

\section{Methods}

\section{Type of Study}

This is a multi-institutional propensity score-matched case-control study, comparing patients undergoing a perioperative management based on the ERAS society principles (Table 1) and control patients undergoing conventional surgical management.

Data were collected in the context of the IMIGASTRIC study and after sharing a specific study protocol.[1] The study was registered at clinical trials.gov with a registration number of NCT02325453.

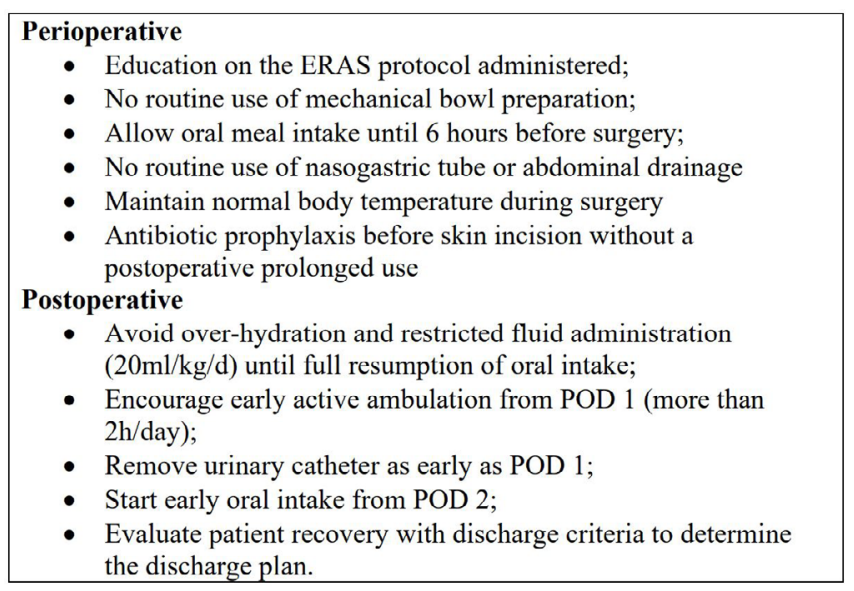

Table 1: ERAS management.

\section{Time Period and Sites}

Data entered into the IMIGASTRIC registry regarding procedures performed until data extraction (November 2019) were analyzed. All involved centers are referral institutions with a well-established gastric cancer program. All diagnostic and surgical interventions at these centers were done according to international guidelines and information stored in institutional prospective data collection systems.

\section{Inclusion criteria}

- Histologically proven gastric cancer

- Preoperative staging work-up performed by upper endoscopy and/or endoscopic ultrasound, and CT scan and in accordance to international guidelines

- Early Gastric Cancer[2, 3]

- Advanced Gastric Cancer[2,3]

- Patients treated with curative intent in accordance to international guidelines $[4,5]$

\section{Exclusion criteria}

- Distant metastases: peritoneal carcinomatosis, liver metastases, distant lymph node metastases, Krukenberg tumors, involvement of other organs

- Patients with high operative risk as defined by the American Society of Anesthesiologists (ASA) score $>4$

- History of previous abdominal surgery for gastric cancer

- Synchronous malignancy in other organs

- Palliative surgery

\section{Data Collection and Reported Outcomes}

This study reported descriptive findings and outcomes among the two groups. Basic patient characteristics, tumor findings, and surgical procedure details were reported. The outcomes section offers a comparison regarding operative results, postoperative recovery, and complications.

\section{Source of Data Analyzed}

Data gathered were obtained from existing records, diagnostic tests, and surgical intervention descriptions. Data were collected and recorded by all institutions through a specific online shared protected system (https://imigastric.logix-software.it/).

The present study was reported in accordance with the STROBE guidelines and statement[6].

\section{Propensity Score Matching}

Propensity score matching analysis was carried out using SPSS software version 23 and R software version 3.1, through the Custom Dialog "PS Matching". Each patient's propensity score was calculated by a multivariable logistic regression model using the covariates of institution, age, sex, comorbidities, body mass index (BMI), surgical approach (open, laparoscopic, robotic) type of gastrectomy, stage of disease.

Patients in the ERAS and Control group were 1:1 matched by the closest propensity score on the logit scale and with a Caliper $=0.2$

\section{Statistics}

IBM SPSS Statistics V.23 was used to carry out the statistical analysis. An intention to treat analysis was performed.

The dichotomous variables were expressed as numbers and percentages, while continuous variables as mean and SD, or median and IQR (minimum and maximum values). Continuous variables were compared using independent $\mathrm{T}$ test.

Pearson's $\chi 2$ test or Fisher's exact test, as appropriate, was used for analysis of categorical data. A P value of 
$<0.05$ was considered statistically significant.

\section{Results}

At the time of this analysis, 1445 patients entered in the IMIGASTRIC registry had information on the ERAS management.

The matching analysis resulted in a total sample of 440 patients, 220 from the ERAS group and 220 from the Control group. The successful matching permitted to obtain a homogeneous distribution of all patient's characteristics (Table 2).

Table 3 shows no significant differences between groups in surgical approach $(\mathrm{P}=0.14)$, type of gastrectomy $(\mathrm{P}=0.31)$, stage of the disease $(\mathrm{P}=0.61)$.

A significantly shorter hospital stay $(\mathrm{P}=0.0004$; Figure 1$)$ was found in the ERAS group versus the Control group (Table 4).

The most relevant benefit was shown in the resumption of a liquid ( $\mathrm{P}=0.01$; Figure 2$)$ and a soft solid diet $(\mathrm{P}=0.007$; Figure 3).

No significant differences in patient mobilization $(\mathrm{P}=0.56)$ and first flatus $(\mathrm{P}=0.07)$ were found.

The ERAS group showed some advantages in the intravenous analgesic use $(\mathrm{P}<0.0001$; Figure 4$)$ than the control group, but no differences were found in the length of antibiotic use $(\mathrm{P}=0.31)$.

In this study the adoption of an ERAS protocol resulted in a significant reduction in post-operative complications $(\mathrm{P}=0.001)$ than the Control group.

\begin{tabular}{|c|c|c|c|}
\hline Variable & ERAS & Control & P value \\
\hline Total no. & 220 & 220 & \\
\hline Age mean \pm SD & $67.14 \pm 13.03$ & $67.18 \pm 12.62$ & 0.97 \\
\hline BMI mean \pm SD & $24.00 \pm 4.18$ & $24.14 \pm 3.04$ & 0.69 \\
\hline Sex no. $(\%)$ & & & 0.77 \\
\hline $\mathrm{M}$ & $132(60)$ & $128(58.2)$ & \\
\hline $\mathrm{F}$ & $88(40)$ & $92(41.8)$ & \\
\hline ASA no. $(\%)$ & & & 0.93 \\
\hline I & $50(22.7)$ & $51(23.2)$ & \\
\hline II & $109(49.5)$ & $114(51.8)$ & \\
\hline III & $61(27.8)$ & $55(25)$ & \\
\hline Comorbidities no. $(\%)$ & $145(65.9)$ & $147(66.8)$ & 0.46 \\
\hline
\end{tabular}

Table 2: Basic patients' characteristics.

\begin{tabular}{|c|c|c|c|}
\hline Variable & ERAS & Control & p value \\
\hline Type of gastrectomy No. (\%) & & & 0.31 \\
\hline Distal & $134(60.9)$ & $140(63.6)$ & \\
\hline Total & $86(39.1)$ & $80(36.4)$ & \\
\hline Surgical approach No. (\%) & & & 0.14 \\
\hline Open & $75(34.1)$ & $87(39.5)$ & \\
\hline MIS & $145(65.9)$ & $133(60.5)$ & \\
\hline Tumor stage No. (\%) & & & 0.61 \\
\hline 0 & $9(4.1)$ & $9(4.1)$ & \\
\hline IA & $39(17.7)$ & $36(16.4)$ & \\
\hline IB & $18(8.2)$ & $17(7.7)$ & \\
\hline IIA & $29(13.2)$ & $32(14.5)$ & \\
\hline IIB & $27(12.3)$ & $24(10.9)$ & \\
\hline IIIA & $25(11.4)$ & $36(16.4)$ & \\
\hline IIIB & $32(14.5)$ & $38(17.3)$ & \\
\hline IIIC & $41(18.6)$ & $28(12.7)$ & \\
\hline
\end{tabular}

Table 3: Surgical and pathological characteristics.

\begin{tabular}{|l|l|l|r|}
\hline \multicolumn{1}{|c|}{ Outcome } & \multicolumn{1}{c|}{ ERAS } & \multicolumn{1}{c|}{ Control } & p value \\
\hline Intraoperative complications no. (\%) & $8(3.6)$ & $10(4.5)$ & 0.81 \\
\hline Intraoperative death no. (\%) & $0(0)$ & $0(0)$ & 1 \\
\hline Hospital stay (days) mean \pm SD & $10.35 \pm 4.76$ & $13.77 \pm 13.61$ & $<0.001$ \\
\hline Mobilization (days) mean \pm SD & $2.37 \pm 1.91$ & $2.65 \pm 6.92$ & 0.56 \\
\hline Liquid diet (days) mean \pm SD & $3.89 \pm 2.7$ & $4.76 \pm 4.41$ & 0.01 \\
\hline Solid diet (days) mean \pm SD & $6.79 \pm 4.91$ & $9.05 \pm 10.87$ & 0.007 \\
\hline Peristalsis (days) mean \pm SD & $2.55 \pm 1$ & $2.59 \pm 1.13$ & 0.67 \\
\hline First flatus (days) mean \pm SD & $3.31 \pm 1.18$ & $3.54 \pm 1.52$ & 0.07 \\
\hline Antibiotic use (days) mean \pm SD & $4.75 \pm 3.86$ & $5.24 \pm 5.83$ & 0.31 \\
\hline Analgesic use (days) mean \pm SD & $2.82 \pm 1.35$ & $4.34 \pm 2.76$ & $<0.001$ \\
\hline Complications no. (\%) & $18(8.2)$ & $43(19.5)$ & 0.001 \\
\hline Complications after discharge no. (\%) & $11(5)$ & $10(4.5)$ & 0.5 \\
\hline
\end{tabular}

Table 4: perioperative outcomes.

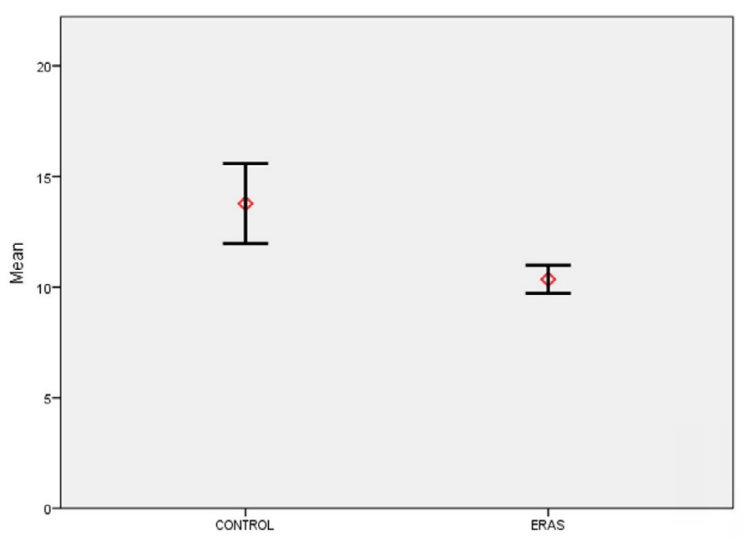

Figure 1: mean difference between groups in the length of hospital stay.

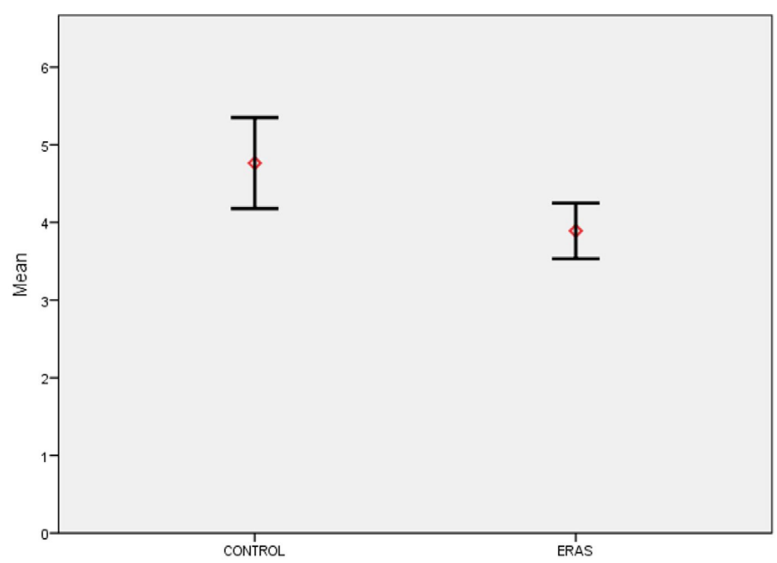

Figure 2: mean difference between groups in starting a liquid intake.

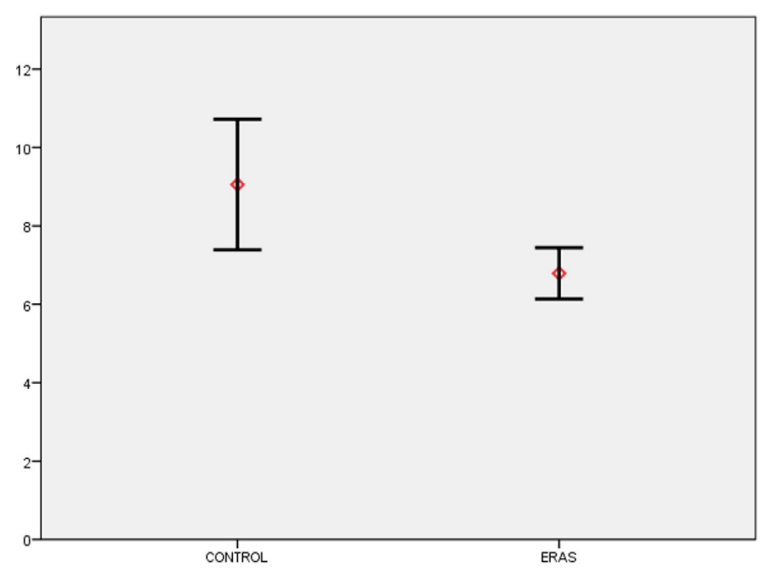

Figure 3: mean difference between groups in starting a soft solid diet. 


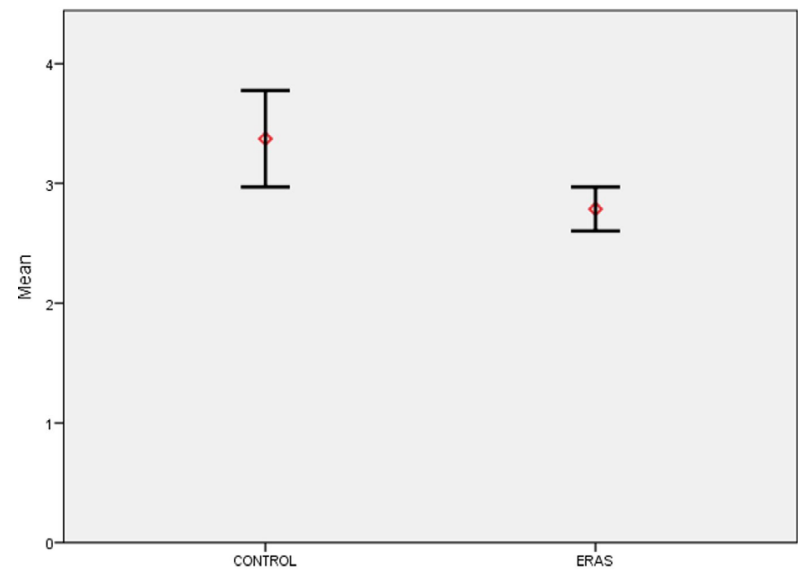

Figure 4: mean difference between groups in the length of intravenous analgesic use.

\section{Discussion}

The present study evaluated the role of the ERAS program in the management of gastric cancer patients using a propensity score case matched analysis to perform a comparison with a conventional care treatment.

A significant decrease in the length of hospital stay and in-hospital postoperative complications were the most relevant findings.

Important items in the ERAS protocol are early mobilization and feeding[7], which is especially facilitated by the absence of the NG tube and drainage, as well as an early removal of the urinary catheter. Smart[8] showed that failure of early patient mobilization is associated with prolonged hospital stay.

Yamada[9] in his study showed that the recovery of bowel function was significantly earlier in the ERAS group than in the conventional group.

In addition, Wang[10] reported that the first day of flatulence after gastric surgery was a mean of one day earlier in patients who received fast-track surgery compared to those who received conventional care.

Some factors such as prolonged fasting and placement of the nasogastric tube appear to cause nausea and contribute to a delay of intestinal recovery[11].

In our study, there was not a significant difference regarding the first flatus. However, we believe that this outcome is subject to a high risk of bias. Instead, variables regarding the oral recovery of food intake can be considered more reliable.

We found a significant advantage in favor of the ERAS group in all steps of food intake, from starting a liquid diet $(3.89 \pm 2.7$ vs $4.76 \pm 4.41 ; \mathrm{P}<0.01)$ to the resumption of a solid diet $(6.79 \pm 4.91$ vs $9.05 \pm 10.87 ; \mathrm{P}=0.007)$.

The ERAS protocols require the patient be not subjected to long periods of fasting.

Early postoperative nutrition reduces postoperative catabolism, accelerates the return of bowel function, and decreases the risk of complications. This was especially studied in colorectal surgery[12,13].

Moreover, Lewis et al.[14] confirmed in their metaanalysis that keeping patients in a fasting state is not beneficial.

Several studies have shown that early oral feeding is feasible and brings benefits in gastric surgery[10, 15]; however, this point remains controversial.

Even if an early postoperative oral feeding has been shown to speed up the recovery after various types of surgery, this approach after gastrectomy has always been seen with suspicion because of some concerns, not actually well demonstrated in the literature, that early food intake may cause anastomotic leakage or intestinal obstruction.

In recent years, several studies have confirmed that early oral food intake after gastric surgery is safe and might be associated with enhanced recovery and shorter hospital stay $[9,16]$.

Particularly, a randomized controlled study has reported data on the safety of early oral feeding starting in the second postoperative day (POD 2) after gastrectomy[17]. The Makuuchi[18] and Pedziwiatr[19] studies, comparing ERAS and conventional management after gastrectomy, have confirmed that oral feeding in POD 2 is safe and allows the reduction of post-operative administration of intravenous fluids and an early discharge[20].

Sugisawa[21] focused on the rate of anastomotic leakage and aspiration pneumonia to evaluate the real risks of early nutrition.

In his study, the incidence of anastomotic leakage was $0.8 \%$, a figure he highlighted as not only lower than that of the subjects in his historical cohort $(1.7 \%)$, but also a result not different or lower (0.8-1.9\%) when compared to previous data in studies in which conventional perioperative care was reported. Therefore, the author concluded that early oral nutrition is not able to adversely affect the anastomotic site. The same results were obtained by Yamada[9, 22], showing a similar incidence in anastomotic leaks (1.1\%).

In our study, the adoption of an ERAS program resulted in a significant reduction in hospital stay with a mean difference of 3.42 days compared to conventional management.

Similar results were obtained by Sugisawa[21] and Wang[10].

In our study, a significant reduction in patients with postoperative complications $(8.2 \%$ versus $19.5 \%$; $\mathrm{P}=0.001$ ) was shown in the ERAS group. Moreover, no differences in readmissions for complications after discharge were shown $(\mathrm{P}=0.5)$.

In conclusion, the adoption of a management based on the ERAS principles for gastric cancer can safely improve the patient's functional recovery, allowing an early discharge and a reduction of overall complications.

\section{Acknowledgements}

None

\section{Contributors}

FG, FF, CC, MC, MP conceptualized and designed the study, acquired, and analyzed data, interpreted the study results, drafted the manuscript, and critically revised the final version of the manuscript.

\section{Funding}

This study received financial support for data extraction and analysis in the context of the Research Startup Projects (2019) granted by "La Sapienza" University of Rome. 


\section{Competing interests}

No benefits in any form have been received or will be received from a commercial party related directly or indirectly to the subject of this article.

\section{Availability of data and materials}

Further information is available from the corresponding author on reasonable request.

\section{Ethics approval}

This study is conducted in compliance with ethical principles originating from the Helsinki Declaration, within the guidelines of Good Clinical Practice and relevant laws/regulations. Trial registration number: NCT02325453.

\section{Provenance and peer review}

Not commissioned; externally peer reviewed.

\section{Open access}

This is an Open Access article distributed in accordance with the Creative Commons Attribution NonCommercial (CC BY-NC 4.0) license, which permits others to distribute, remix, adapt, build upon this work noncommercially, and license their derivative works on different terms, provided

the original work is properly cited and the use is non-commercial. See: http://creativecommons.org/ licenses/by-nc/4.0/

\section{References}

[1] Desiderio J, Jiang ZW, Nguyen NT, Zhang S, Reim D, Alimoglu O, et al. Robotic, laparoscopic and open surgery for gastric cancer compared on surgical, clinical and oncological outcomes: a multi-institutional chart review. A study protocol of the International study group on Minimally Invasive surgery for GASTRIc Cancer-IMIGASTRIC. BMJ open. 2015;5:e008198.

[2] Japanese classification of gastric carcinoma: 3rd English edition. Gastric Cancer. 2011;14:101-12.

[3] Murakami T. Pathomorphological diagnosis. Definition and gross classification of early gastric cancer. Gann Monogr Cancer Res. 1971:53-5.

[4] Japanese gastric cancer treatment guidelines 2010 (ver. 3). Gastric Cancer. 2011;14:113-23.

[5] Waddell T, Verheij M, Allum W, Cunningham D, Cervantes A, Arnold D. Gastric cancer: ESMO-ESSO-ESTRO clinical practice guidelines for diagnosis, treatment and follow-up. European journal of surgical oncology : the journal of the European Society of Surgical Oncology and the British Association of Surgical Oncology. 2014;40:584-91.

[6] von Elm E, Altman DG, Egger M, Pocock SJ, Gotzsche PC, Vandenbroucke JP, et al. The Strengthening the Reporting of Observational Studies in Epidemiology (STROBE) Statement: guidelines for reporting observational studies. International journal of surgery. 2014;12:1495-9.

[7] Vlug MS, Wind J, Hollmann MW, Ubbink DT, Cense HA, Engel $\mathrm{AF}$, et al. Laparoscopy in combination with fast track multimodal management is the best perioperative strategy in patients undergoing colonic surgery: a randomized clinical trial (LAFA-study). Ann Surg. 2011;254:868-75

[8] Smart NJ, White P, Allison AS, Ockrim JB, Kennedy RH, Francis NK. Deviation and failure of enhanced recovery after surgery following laparoscopic colorectal surgery: early prediction model. Colorecta disease : the official journal of the Association of Coloproctology of Great Britain and Ireland. 2012;14:e727-34.

[9] Yamada T, Hayashi $T$, Cho $H$, Yoshikawa $T$, Taniguchi $H_{\text {, }}$ Fukushima $\mathrm{R}$, et al. Usefulness of enhanced recovery after surgery protocol as compared with conventional perioperative care in gastric surgery. Gastric Cancer. 2012;15:34-41.

[10] Wang D, Kong Y, Zhong B, Zhou X, Zhou Y. Fast-track surgery improves postoperative recovery in patients with gastric cancer: a randomized comparison with conventional postoperative care. Journal of gastrointestinal surgery : official journal of the Society for Surgery of the Alimentary Tract. 2010;14:620-7.

[11] Teeuwen PH, Bleichrodt RP, Strik C, Groenewoud JJ, Brinkert W, van Laarhoven CJ, et al. Enhanced recovery after surgery (ERAS) versus conventional postoperative care in colorectal surgery. Journal of gastrointestinal surgery : official journal of the Society for Surgery of the Alimentary Tract. 2010;14:88-95.

[12] Lewis SJ, Egger M, Sylvester PA, Thomas S. Early enteral feeding versus "nil by mouth" after gastrointestinal surgery: systematic review and meta-analysis of controlled trials. Bmj. 2001;323:773-6.

[13] Han-Geurts IJ, Hop WC, Kok NF, Lim A, Brouwer KJ, Jeekel J. Randomized clinical trial of the impact of early enteral feeding on postoperative ileus and recovery. The British journal of surgery. 2007;94:555-61.

[14] Lewis SJ, Andersen HK, Thomas S. Early enteral nutrition within $24 \mathrm{~h}$ of intestinal surgery versus later commencement of feeding: a systematic review and meta-analysis. Journal of gastrointestinal surgery : official journal of the Society for Surgery of the Alimentary Tract. 2009;13:569-75.

[15] Jo DH, Jeong O, Sun JW, Jeong MR, Ryu SY, Park YK. Feasibility study of early oral intake after gastrectomy for gastric carcinoma. Journal of gastric cancer. 2011;11:101-8.

[16] Scatizzi M, Kroning KC, Boddi V, De Prizio M, Feroci F. Fast-track surgery after laparoscopic colorectal surgery: is it feasible in a general surgery unit? Surgery. 2010;147:219-26.

[17] Hur H, Kim SG, Shim JH, Song KY, Kim W, Park CH, et al. Effect of early oral feeding after gastric cancer surgery: a result of randomized clinical trial. Surgery. 2011;149:561-8.

[18] Makuuchi R, Sugisawa N, Kaji S, Hikage M, Tokunaga M, Tanizawa $Y$, et al. Enhanced recovery after surgery for gastric cancer and an assessment of preoperative carbohydrate loading. Eur J Surg Oncol. 2016.

[19] Pedziwiatr M, Matlok M, Kisialeuski M, Migaczewski M, Major $\mathrm{P}$, Winiarski M, et al. Short hospital stays after laparoscopic gastric surgery under an Enhanced Recovery After Surgery (ERAS) pathway: experience at a single center. European surgery : ACA : Acta chirurgica Austriaca. 2014;46:128-32.

[20] Terashima M. The earlier the better? Gastric Cancer. 2014;17:197-9. [21] Sugisawa N, Tokunaga M, Makuuchi R, Miki Y, Tanizawa Y, Bando E, et al. A phase II study of an enhanced recovery after surgery protocol in gastric cancer surgery. Gastric Cancer. 2016;19:961-7.

[22] Yamada T, Hayashi T, Aoyama T, Shirai J, Fujikawa H, Cho H, et al. Feasibility of enhanced recovery after surgery in gastric surgery: a retrospective study. BMC surgery. 2014;14:41. 